
Not for reproduction, distribution or commercial use.

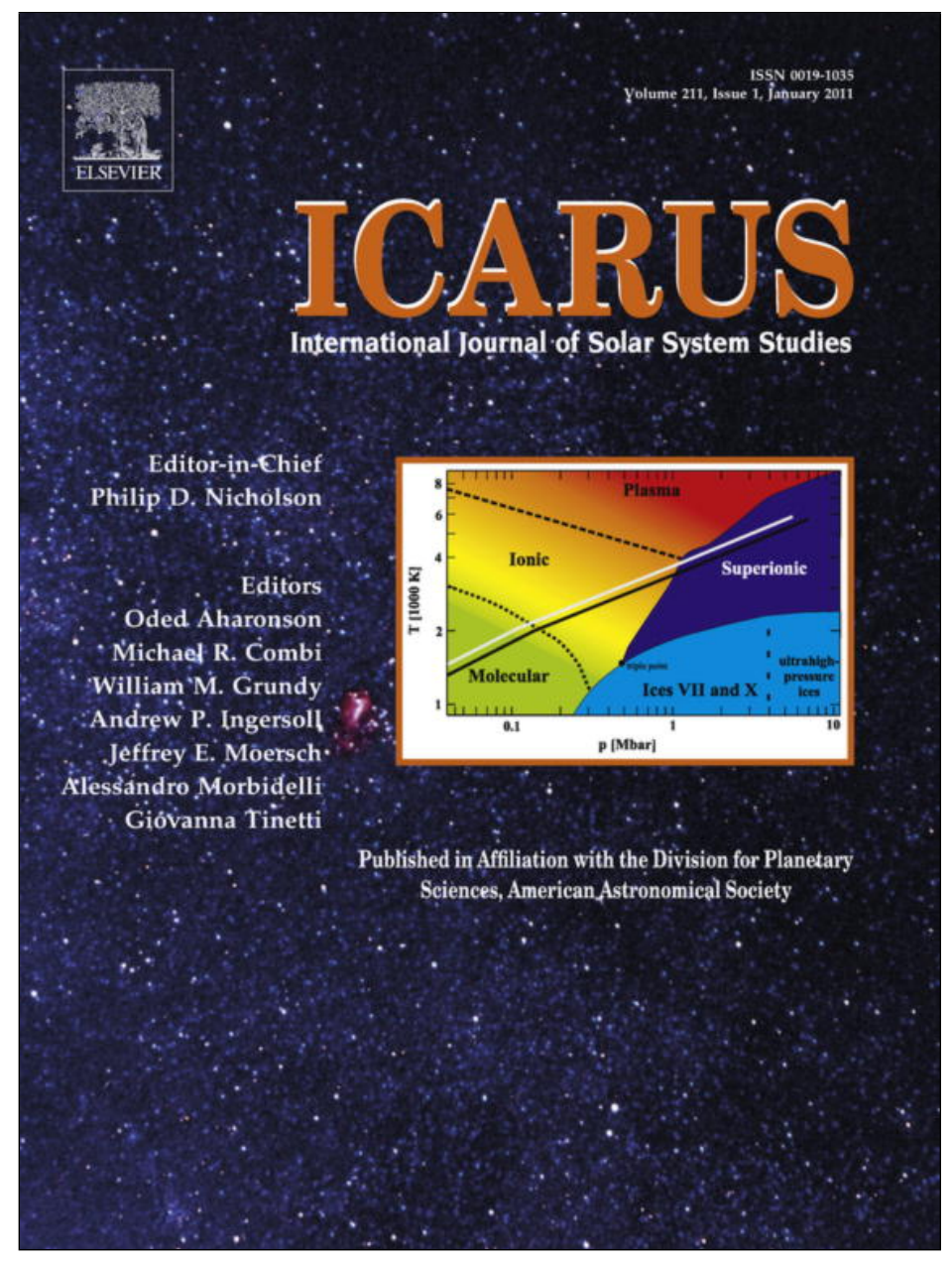

This article appeared in a journal published by Elsevier. The attached copy is furnished to the author for internal non-commercial research and education use, including for instruction at the authors institution and sharing with colleagues.

Other uses, including reproduction and distribution, or selling or licensing copies, or posting to personal, institutional or third party websites are prohibited.

In most cases authors are permitted to post their version of the article (e.g. in Word or Tex form) to their personal website or institutional repository. Authors requiring further information regarding Elsevier's archiving and manuscript policies are encouraged to visit:

http://www.elsevier.com/copyright 


\title{
Solar radiation pressure on (99942) Apophis
}

\author{
J. Žižka ${ }^{\mathrm{a}, \mathrm{b}}, \mathrm{D}$. Vokrouhlický ${ }^{\mathrm{a}, *}$ \\ ${ }^{a}$ Institute of Astronomy, Charles University, V Holešovičkách 2, 18000 Prague 8, Czech Republic \\ ${ }^{\mathrm{b}}$ Nicholas Copernicus Observatory and Planetarium, Kraví hora 2, 61600 Brno, Czech Republic
}

\section{A R T I C L E I N F O}

\section{Article history:}

Received 9 April 2010

Revised 12 August 2010

Accepted 18 August 2010

Available online 24 August 2010

\section{Keywords:}

Celestial mechanics

Asteroids, Dynamics

\begin{abstract}
A B S T R A C T
Near-Earth asteroid (99942) Apophis currently resides among the top positions on the list of objects with small, yet non-zero impact probability with the Earth. For that reason an unusual observational and theoretical effort has been dedicated to precisely characterize its future orbit. Here we discuss orbital perturbation of Apophis due to incident and reflected solar radiation pressure (SRP). We both revisit recent analytical estimate of the SRP effects for this body and also formulate a numerical approach allowing us to compute the SRP orbital perturbation under general assumptions. Contrary to some previous results, we show that SRP has a much smaller effect on the Apophis trajectory than does the thermal re-radiation force which produces the Yarkovsky effect. When the Yarkovsky effect becomes constrained enough in the future, our approach may be used to improve the orbit determination for this asteroid.

(c) 2010 Elsevier Inc. All rights reserved.
\end{abstract}

\section{Introduction}

Near-Earth asteroids present a threat by their possible impacts onto the Earth (e.g., Chesley et al., 2002; Chapman, 2004). Accurate orbital ephemeris and the impact likelihood analysis represent a starting point for further considerations such as mitigation actions. When the identified impact possibility is distant in future (i.e. tens of years and more), a very accurate orbital model, beyond the usual needs of the asteroid orbit computations, is required to analyze the problem. Vokrouhlický et al. (2000) and Vokrouhlický and Milani (2000) pointed out that several forces of non-gravitational origin may be needed for this task. The Yarkovsky effect due to the recoil of thermally re-radiated sunlight (e.g., Bottke et al., 2002, 2006) is the most important of them. Indeed, search efforts have detected this effect acting on several near-Earth asteroids (e.g., Chesley et al., 2003, 2008; Vokrouhlický et al., 2008) and detailed studies of orbital evolution for several potentially hazardous objects has identified the Yarkovsky effect as the most significant to obscure future position predictions (e.g., Giorgini et al., 2002, 2008; Chesley, 2006; Milani et al., 2009).

Asteroid (99942) Apophis, a former record-holder in the impact threat scale, has an unusually close approach to the Earth in April 13,2029 . At that date, it will pass some $38,000 \mathrm{~km}$ from the Earth center and subsequently will be perturbed by the Earth's gravity into a new heliocentric orbit, switching from the Aten to the Apollo category (Fig. 1). In fact, soon after its discovery in December 2004, Apophis was initially estimated to have an impact probability

\footnotetext{
* Corresponding author.

E-mail addresses: jinziz@centrum.cz (J. Žižka), vokrouhl@cesnet.cz (D. Vokrouhlický).
}

larger than any prior case (e.g., Chesley, 2006), but was quickly reduced in magnitude and pushed to the mid 2030s as additional measurements were reported (Chesley, 2006; Chesley et al., 2009; Giorgini et al., 2008). This is because a number of resonant return-orbits are within (or very close to) the orbital uncertainty region during the 2029 approach. Even if Apophis eventually does not impact the Earth, the high-accuracy orbit determination efforts developed for this complicated case will certainly be applicable to similar cases in the future.

A thorough analysis of the Apophis orbit, including its discovery circumstances and improvements of the orbital determination as a function of increasing observation dataset, has been published by Chesley (2006) and Giorgini et al. (2008). While both took into account the effects of the thermal forces (the Yarkovsky effect), perturbations due to the solar radiation pressure in optical waveband have been considered by the latter only. Using a very simple, but in fact satisfactory zero-order approximation in the numerical integration, Giorgini et al. (2008) concluded that the solar radiation pressure effect on Apophis is much less than the Yarkovsky effect. By contrast, Rubincam (2007) performed a specific analysis of the radiation pressure orbital effects for Apophis and concluded they can be as significant as those due to the thermal forces (the Yarkovsky effect). The contradictory conclusions from these two works ${ }^{1}$ motivated us to take a closer look at the effects of the solar radiation pressure in the orbit of Apophis.

\footnotetext{
${ }^{1}$ Note that Giorgini et al. (2008) were aware of a preprint version of Rubincam (2007) and noted the difference between their numerical results and those of Rubincam. They assumed the extreme shape adopted by Rubincam (2007) made the radiation pressure effect unrealistically large.
} 


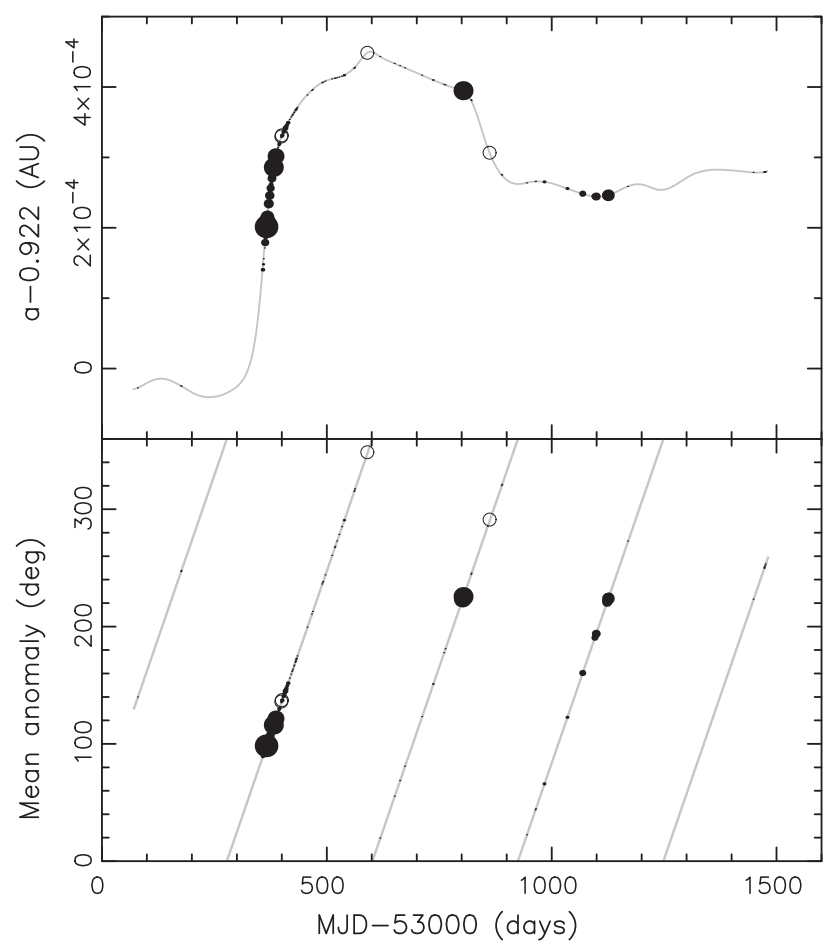

Fig. 1. Osculating semimajor axis (top) and mean anomaly (bottom) for the orbit of (99942) Apophis from 2004.0 till 2008.2 (grey curve). Symbols show where observations have been obtained so far and collected into 1-day bins; size of the symbol is scaled by number of observations acquired during that day. Full circles for optical astrometry, open circles for radar astrometry. Majority of observations (some 60\%) was obtained during the 2004-2005 close approach. The significant increase of the heliocentric semimajor axis seen in December 2004 is due to the close approach to the Earth; a much more spectacular change will occur during the 400 times closer approach in April 2029, when the semimajor axis will increase to $\sim 1.1 \mathrm{AU}$.

The theory of radiation force for an arbitrarily-shaped asteroid is given in Section 2 and its numerical implementation in Section 3. Results of our numerical simulations are summarized in Section 4. Section 2 also contains a minor correction of the analytical computations of Rubincam (2007).

\section{Theory}

Solar radiation impinging the surface of an asteroid is removed from the incident beam and reprocessed in two ways: (i) the first part, included in this paper, is directly scattered in the optical waveband (since the dominant part of sunlight is in optical) and (ii) the second part, not included in this paper, is absorbed and re-emitted in thermal waveband. The second part, namely the recoil acceleration due to thermal radiation, results in what is known as the Yarkovsky effect (e.g., Bottke et al., 2002, 2006).

The prime goal of our work is the accuracy of the solar radiation pressure computation. For that reason we do not restrict to (i) a simplest Lambertian reflection of the sunlight and (ii) a simplest possible geometric shapes of the asteroid (such as spherical or ellipsoidal), but instead we keep the formulation as general as possible. Such an approach is handled numerically rather than analytically, and requires that the fundamental level at which we describe the radiation pressure involves momentum budget for an infinitesimal surface facet upon which some fraction of the incident sunlight is absorbed, the rest scattered through reflection. The total effect is then obtained by numerical integration over all surface facets.

\subsection{Incident sunlight}

The dynamical effect of the incident sunlight arises simply by virtue of its removal from the solar radiation flux as a momentum transfer to the object. It is thus given by (e.g., Vokrouhlický and Milani, 2000)

$\mathbf{f}_{\text {inc }}=-\frac{F S_{\perp}}{m c} \mathbf{n}_{0}$,

where $F$ is the sunlight flux at the asteroid heliocentric distance, $S_{\perp}$ is the instantaneous cross-section of the asteroid with respect to the solar radiation, $m$ is the asteroid mass, $c$ the velocity of light and $\mathbf{n}_{0}$ is the instantaneous unit vector directed from the asteroid to the Sun. Note $\mathbf{f}_{\text {inc }}$ is radially directed acceleration with two primary sources of temporal variability: (i) the flux $F$ varies along an eccentric orbit as the heliocentric distance $d$ changes $\left(F \propto d^{-2}\right)$ and (ii) the cross-section $S_{\perp}$ changes due to asteroid rotation and its revolution about the Sun for an irregularly-shaped body. If only the first phenomenon existed, the dynamical effect of the impinging sunlight would be equivalent to redefining the orbital elements due to effectively smaller value of Sun's gravitational mass (e.g., Dermott et al., 2001). However, the cross-section $S_{\perp}$ variation slightly complicates the situation and adds short-period perturbations that needs to be modeled numerically. At each timestep of the integrator we thus need to evaluate

$S_{\perp}=\int_{S_{0}}\left(d \mathbf{S} \cdot \mathbf{n}_{0}\right) \Sigma\left(\mathbf{R}, \mathbf{n}_{0}\right)$,

where the integration is performed over the illuminated portion $S_{0}$ of the asteroid surface, $d \mathbf{S}$ is an outward-oriented surface element and $\Sigma$ is the shadow function. ${ }^{2}$ The latter is either 1 , if the surface facet is illuminated, or 0 , if the surface facet is shadowed by other parts of the asteroid; $\Sigma$ is a function of $\mathbf{n}_{0}$, the transformation matrix $\mathbf{R}$ from the ecliptic inertial system to the body-frame system, and the overall shape model. An effective analysis of $\Sigma$ is not a trivial task for highly irregular shapes. Since we have the asteroid shape models available as discrete polyhedrons (with typically thousands of facets), we replace the integration in (2) by summation over the surface facets. The mutual shadowing conditions are treated with a rather inefficient " $N{ }^{2}$ method" described in Appendix B.2 of Čapek (2007) (available through http://sirrah.troja.mff.cuni.cz/ davok/). The procedure basically inspects each of the $N$ surface elements and, given the instantaneous solar position, seeks whether it projects a shadow on another surface element. The algorithm was somewhat accelerated by proper sorting of the elements, such that they are listed according to their increasing distance from the asteroid's centerof-mass and thus the origin of the body-centered reference frame.

\subsection{Sunlight scattered on the asteroid surface}

We now turn to discuss the dynamical effects of the sunlight scattered by the asteroid surface. Assume an infinitesimal surface facet $d \mathbf{S}=\mathbf{N} d S$ with outward normal vector $\mathbf{N}$. Let $\mathbf{N}_{0}$ denote unit vector of the local direction to the Sun and choose an arbitrary unit vector $\mathbf{N}_{\text {ray }}$ directed to the hemisphere above the given surface element. The specific radiation intensity $I$ of the scattered sunlight along $\mathbf{N}_{\text {ray }}$ is given by

$I=I\left(\mathbf{N}_{0}, \mathbf{N}_{\text {ray }}\right)=\operatorname{Fr}\left(\mathbf{N}_{0}, \mathbf{N}_{\text {ray }}\right)$,

\footnotetext{
${ }^{2}$ Here we formally define $S_{0}$ as a sample of surface facets for which $d \mathbf{S} \cdot \mathbf{n}_{0}>0$, a necessary condition for illumination. The complete analysis of the facet illumination requires $\Sigma=1$ to make sure other parts of the surface do not produce a shadow at the location of $d \mathbf{S}$. We display these two conditions explicitly to describe the complexity of the illumination condition.
} 
where $F$ is the incident radiation flux and $r\left(\mathbf{N}_{0}, \mathbf{N}_{\text {ray }}\right)$ is the bidirectional reflectance function (e.g., Mihalas, 1978). Assuming that $r$ is a macroscopic quantity, that characterizes reflectance of random sample of microscopic scatterers, we have a reduced dependence $r=r\left(\mu, \mu_{0}, \mathbf{N}_{0} \cdot \mathbf{N}_{\text {ray }}\right)$. Here $\mu_{0}=\mathbf{N}_{0} \cdot \mathbf{N}$ and $\mu=\mathbf{N}_{\text {ray }} \cdot \mathbf{N}$ are directional cosines of the solar position and scattered light-ray direction with respect to the surface normal $\mathbf{N}$. It is also convenient to introduce local reference system with the $z$-direction along $\mathbf{N}$ and $x$-direction along

$\mathbf{M}=\frac{\mathbf{N}_{0}-\mu_{0} \mathbf{N}}{\sqrt{1-\mu_{0}^{2}}}$.

With that choice, and spherical coordinates $(\theta, \phi)(\mu=\cos \theta)$, we can write

$\mathbf{N}_{0}=\left(\begin{array}{c}\sqrt{1-\mu_{0}^{2}} \\ 0 \\ \mu_{0}\end{array}\right)$

and

$\mathbf{N}_{\text {ray }}=\left(\begin{array}{c}\sqrt{1-\mu^{2}} \cos \phi \\ \sqrt{1-\mu^{2}} \sin \phi \\ \mu\end{array}\right)$

In this way, we have

$\cos \alpha=\mathbf{N}_{0} \cdot \mathbf{N}_{\text {ray }}=\mu \mu_{0}+\sqrt{1-\mu^{2}} \sqrt{1-\mu_{0}^{2}} \cos \phi$

for the cosine of mutual angle $\alpha$ between $\mathbf{N}_{0}$ and $\mathbf{N}_{\text {ray. }}$.

Many studies of the sunlight reflection on the solid surfaces in the Solar System used Hapke's class of models. Since we do not need the highest accuracy in our work, we adopt Hapke's bidirectional reflectance function in a single-scattering approximation (e.g., Hapke, 1981, 2002)

$r\left(\mu, \mu_{0}, \phi\right)=\frac{w}{4 \pi} \frac{\mu_{0}}{\mu+\mu_{0}}\left[\left(1+B_{0} B_{s}(\alpha)\right) P(\alpha)+H(\mu) H\left(\mu_{0}\right)-1\right]$,

where $B_{s}(\alpha)$ is the backscatter function which describes the opposition effect of the surface

$B_{s}(\alpha)=\left[1+\left(1 / h_{s}\right) \tan (\alpha / 2)\right]^{-1}$,

$H(x)$ is the Chandrasekhar's $H$-function, approximated in our computations with $(\gamma=\sqrt{1-w})$

$H(x)=\frac{1+2 x}{1+2 \gamma x}$

and $P(\alpha)$ describes angular scattering properties of a single (average) surface particle. Here we consider the case of HenyeyGreenstein function

$P(\alpha)=\frac{1-g^{2}}{\left(1+2 g \cos \alpha+g^{2}\right)^{3 / 2}}$,

with an anisotropy parameter $g$. We note that such a simplified Hapke model involves the following free parameters: (i) $w$ the average single-scattering albedo, (ii) $B_{0}$ the opposition surge amplitude parameter, (iii) $h_{s}$ characterizes the width of opposition surge, and (iv) $g$ the asymmetry factor of a single-particle phase function (Henyey-Greenstein parameter). A number of authors derived their values from a high-quality photometry of individual asteroids and/ or derived mean values for a given spectroscopic class (e.g., Helfenstein and Veverka, 1989). We shall use this general information, since the Apophis photometry is not prolific enough to derive specific value of $\left(w, B_{0}, h_{s}, g\right)$ parameters for this object.
Before dealing with the recoil acceleration due to the scattered sunlight, we discuss a couple of useful parameters related to the scattering law described above. Most importantly we mention various definitions of "albedo" parameter. The hemispheric albedo $A_{h}$ defines how the incident sunlight energy is partitioned between the reflection in optical wavelengths and thermal diffusion into the body. It is given by

$A_{h}\left(\mu_{0}\right)=\frac{1}{\mu_{0}} \int_{\Omega_{+}} d \Omega \mu r\left(\mu, \mu_{0}, \phi\right)$,

where $\Omega_{+}$denotes the upper hemisphere on a unit sphere such that the integration in (12) goes as $\int_{\Omega_{+}} d \Omega=\int_{0}^{1} d \mu \int_{0}^{2 \pi} d \phi$. With $A_{h}\left(\mu_{0}\right)$ defined as above, the $F \mu_{0} A_{h}\left(\mu_{0}\right)$ amount of radiation energy is directly scattered and $F \mu_{0}\left[1-A_{h}\left(\mu_{0}\right)\right]$ amount of radiation energy is thermally reprocessed. The second concept is that of geometric (or physical) albedo $A_{g}$ which gives the ratio of brightness of a sphere observed at zero phase to the brightness of a Lambert disk. We have

$A_{g}=2 \pi \int_{0}^{1} \mu_{0} r\left(\mu_{0}, \mu_{0}, 0\right) d \mu_{0}$,

which for the above given Hapke's model (8) yields

$A_{g}=\frac{1}{2} r_{0}\left(1+\frac{1}{3} r_{0}\right)+\frac{w}{8}\left[\left(1+B_{0}\right) P(0)-1\right]$,

with $r_{0}=(1-\gamma) /(1+\gamma)$ (e.g., Hapke, 1981). Photometric observations of Delbò et al. (2007) yield $A_{g}=0.33 \pm 0.04$ for (99942) Apophis. Eq. (14) thus provides a correlated constraint on the Hapke parameters of the Apophis surface reflectivity. Finally, we have Bond's albedo $A_{B}$, given by

$A_{B}=2 \int_{0}^{1} \mu_{0} A_{h}\left(\mu_{0}\right) d \mu_{0}$,

which is the total amount of energy scattered by a spherical object in all directions to the energy of the incident sunlight. Still more involved definitions of the "albedo values" may be needed for the analytic radiation force computation: for instance a second-order moment of the hemispheric albedo is the required quantity when dealing with radiation diffusion on a sphere (e.g., Vokrouhlický and Bottke, 2001).

Turning now to the dynamical effect of the reflected sunlight, we note that the infinitesimal recoil acceleration exerted on the surface facet $d S$ is given by (e.g., Mihalas, 1978)

$d \mathbf{f}_{\text {sca }}=-\sum \frac{d S}{m c} \int_{\Omega_{+}} d \Omega \mu \mathbf{N}_{\text {ray }} I\left(\mathbf{N}_{0}, \mathbf{N}_{\text {ray }}\right)$

(recall $\Sigma$ is the shadow function from Eq. (2)). With Hapke's model introduced above, we have

$d \mathbf{f}_{\text {sca }}=-\sum \frac{F}{m c}\left(K_{1} \mathbf{N}+K_{2} \mathbf{M}\right) d S$,

where

$K_{1}\left(\mu_{0}\right)=\int_{\Omega_{+}} d \Omega \mu^{2} r\left(\mu, \mu_{0}, \phi\right)$,

$K_{2}\left(\mu_{0}\right)=\int_{\Omega_{+}} d \Omega \mu \sqrt{1-\mu^{2}} \cos \phi r\left(\mu, \mu_{0}, \phi\right)$.

Note that both $K_{1}$ and $K_{2}$ coefficients depend on the cosine $\mu_{0}$ of the local solar zenith angle and should be evaluated at each integration timestep for each facet. We prevented too large computer time requirements by precomputing $K_{1}$ and $K_{2}$ for a sufficiently dense grid of $\mu_{0}$-values and then used linear interpolation for evaluation of the infinitesimal recoil contributions (17). An example of the $K_{1}$ and $K_{2}$ function dependence on $\mu_{0}$ is shown in Fig. 2 . 


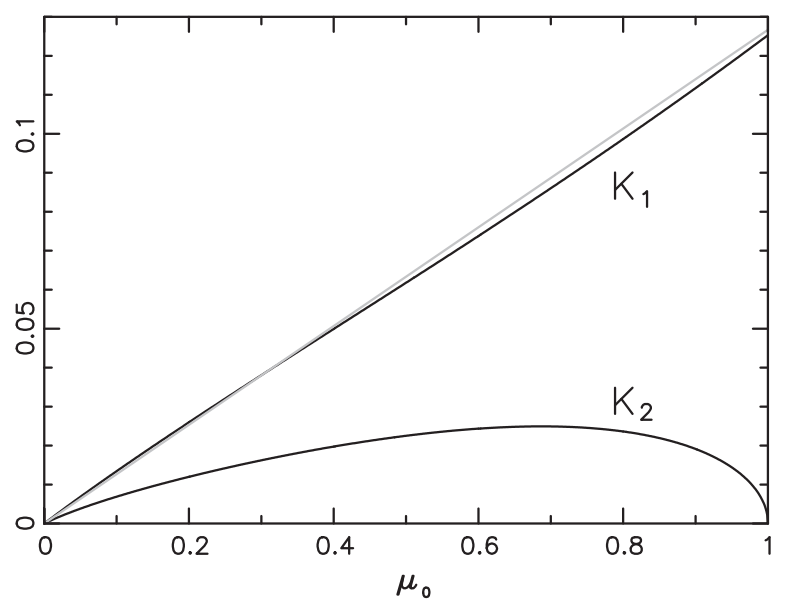

Fig. 2. $K_{1}\left(\mu_{0}\right)$ and $K_{2}\left(\mu_{0}\right)$ functions computed for Hapke parameters $w=0.5$, $g=-0.35, h_{s}=0.02$ and $B_{0}=0.97$ used in Section 4. Note the $K_{1}\left(\mu_{0}\right)$ function is well approximated with $2 A_{B} \mu_{0} / 3$ shown by the grey line $\left(A_{B} \simeq 0.2\right.$ is the Bond albedo from Eq. (15)).

The total recoil acceleration of the asteroid is formally given by integrating (17) over the illuminated surface $S_{0}$ :

$\mathbf{f}_{\mathrm{sca}}=\int_{S_{0}} d \mathbf{f}_{\mathrm{sca}}$.

In practice though, we use a discrete model of the asteroid shape consisting of a finite number of surface facets (usually several thousands). Integration in (20) is then replaced with summation over the model facets.

For sake of comparison with previous work we also note that the case of Lambertian diffusion on the surface is characterized with

$r\left(\mu, \mu_{0}, \phi\right)=\frac{\mu_{0}}{\pi} A$,

where $A$ is a single "albedo" parameter in this model, equal to its hemispheric and Bond values. From (18) and (19) we easily obtain $K_{1}=\frac{2}{3} A \mu_{0}$ and $K_{2}=0$ in this case.

\subsection{Rubincam's hemispheric model}

While we resort to an entirely numerical approach below, see Sections 3 and 4, we find it interesting to comment on the model presented in Rubincam (2007). Rubincam performed analytical computation of the radiation recoil force due to isotropically scattered sunlight on a body of particularly simple shape, namely a hemisphere. Inserting this effect into Gauss equations, Rubincam obtained an estimate of the time-averaged perturbation of orbital elements and from there he drew conclusions about the magnitude of the effect of radiation pressure on the Apophis trajectory. He correctly focused on secular effects in the semimajor axis value $\overline{(d a / d t)}$, which have a potential to produce the most significant in-orbit displacement, and noted that only the scattered sunlight yields $\overline{(d a / d t)} \neq 0$. Estimating its value for the assumed parameters of Apophis, Rubincam concluded that the radiation pressure may contribute in an important way to the orbit uncertainty budget during the 2029 encounter, noting in particular that it can be comparable to the effects due to the thermal forces (the Yarkovsky effect).

While this work represents an interesting attempt, we show here that its conclusion is incorrect for three separate reasons. First, the assumed hemispheric shape in Rubincam (2007) stretches the effect too much as already guessed by Giorgini et al. (2008). In a more detailed approach (Section 4), when we use a set of reasonable asteroid shapes in our numerical implementation, we obtain an effect order of magnitude smaller than predicted with the hemispheric model. Second, Rubincam's analytic calculation contains a small mistake that makes the effect apparently larger than it actually is. Finally, we should also point out that Rubincam's estimate has a conceptual flaw of folding the thermal (Yarkovsky) part of the perturbation into the reflected radiation budget (by using an albedo equal to unity). In fact, when properly modeled the orbital effect of the thermal forces with a non-zero value of the surface thermal inertia may be quite larger than the corresponding effect of the reflected radiation. As a result, one has to carefully distinguish the radiative orbital effects in optical and thermal in a proportion given by the albedo value.

Leaving the numerical experiments to Section 4, we now comment on the analytic results in Rubincam (2007). Basically all of them are performed carefully, but the final result suffers a small omission. Using the notation in that paper, the orbit-averaged semimajor axis drift in Eq. (33) of Rubincam (2007) should have read

$\frac{\overline{d a}}{d t}=-\frac{2(3 \pi-8)}{3 \pi^{2} n} \frac{F_{\mathrm{E}}}{c \rho R_{\mathrm{A}}} \frac{e}{1-e^{2}}\left(\frac{a_{0}}{a}\right)^{2} \sin \beta$.

Note the factor $\pi^{2}$ in the denominator, rather than $\pi$ in Rubincam's result. Inserting the orbital parameters of Apophis we obtain $\overline{d a / d t} \simeq-35 \sin \beta \mathrm{m} / \mathrm{y}$, three times smaller than Rubincam's estimate (Eq. (36); where $\beta$ is the angle between the hemisphere axis and direction to the apocentre in Rubincam's model). As a result, the maximum along-track displacement due to the radiation pressure in Rubincam's model becomes $\sim \pm 80 \mathrm{~km}$.

However, in the next sections we demonstrate that at all likelihood the true effect of the radiation pressure is yet another order of magnitude smaller than this estimate. In this respect one has to note that the large effect in Rubincam's model is a direct result of an extreme north-south shape asymmetry in the hemisphere model. Any more symmetric model would provide smaller effect; for instance, a spheroidal model evaluated also using analytic means by Vokrouhlický and Milani (2000) yields basically zero effect.

\section{Numerical model and simulations}

We implemented computation of the radiation pressure acceleration $\mathbf{f}_{\mathrm{SRP}}=\mathbf{f}_{\text {inc }}+\mathbf{f}_{\mathrm{sca}}$ into the orbit determination software 0rbFit provided by the University of Pisa dynamical group. ${ }^{3}$ An arbitrary shape model is assumed and represented by a polyhedron with a large number of surface facets. Radar imaging and lightcurve analysis has not resulted in a shape model and pole orientation for (99942) Apophis yet; very likely, both will be obtained during its close approach to Earth in January 2013 (e.g., Giorgini et al., 2008). In this situation, the best we can do is to consider known shape models for near-Earth asteroids as a possible template of the Apophis shape and test the orbital effects due to the radiation pressure using these models. Since these are still not numerous enough, we also use a sample of artificial shape models known as Gaussian random spheres whose parameters have been calibrated by the shape models of the main belt asteroids (see Muinonen, 1998; Muinonen and Lagerros, 1998; Vokrouhlický and Čapek, 2002). The pole position is considered random in space, and the parameters of the bidirectional

\footnotetext{
3 The OrbFit software can be downloaded from http://adams.dm.unipi.it/ orbmaint/orbfit/. We used a high-accuracy 15th order Radau-Everhart integrator included in this package to propagate orbit of Apophis and do not integrate past the very close 2029 Earth encounter. This way, accumulation of the integrator errors should not play an important role (see Giorgini et al., 2008). We should point out that our work is not a part of general distribution of OrbFit but may be requested from the authors.
} 
reflectivity function (or just the albedo value in Lambertian approximation) are assumed in accord with the known geometric albedo value $A_{g}=0.33 \pm 0.04$. Apophis rotation period has been constrained, though not accurately determined as yet, by the lightcurve observations. We take the $\sim 30.5 \mathrm{~h}$ value by Behrend et al. (2005).

With the lack of detailed information (such as the shape, pole and reflectivity parameters) we cannot compute the radiation pressure orbital displacement for Apophis accurately. Our approach is to compute it for a large sample of shape, pole and reflectivity-parameters possibilities and characterize the results in a statistical way using a mean/median value and a standard deviation. This defines a given computer run. In each individual simulation of a run, i.e. particular choice of the shape, pole and reflectivity parameters, we first perform an orbit determination for Apophis using all available observations, both sky-plane and radar astrometry (Fig. 1). The best-fit orbit is numerically propagated to the close approach in April 2029, and the geocentric state vector compared to the prediction of the nominal, conservative model where the effects of the radiation forces were not included. ${ }^{4}$ The nominal model contains all relevant perturbations necessary for an accurate orbit determination, namely all planetary perturbations (including lunar effects) and the relativistic effects. None of the seven radar observations is rejected, and only four out of 1399 optical observations are rejected and considered as outliers. The root mean square of the residuals is only $\sim 0.26$ arcsec for all models in the run. In particular, because the available observations cover a short interval of time, from March 2004 till January 2008, the quality of the fit using the nominal model and the extended model by the radiation forces is the same. ${ }^{5}$

\section{Results}

In this section we perform a number of simulations with the goal to reliably estimate the importance of the radiation pressure effects for the foreseeable Apophis' ephemerides, focusing on the orbital displacement during the very close approach in April 2029. We progress step-by-step starting with simpler cases and proceed to more complicated ones later.

\subsection{Spherical model}

At first, we considered the simplest shape model of the asteroid, namely a sphere. Sunlight scattering is modeled using the Lambertian model. In this case, we used two possibilities to implement the solar radiation pressure effects: (i) direct evaluation of the radiation pressure acceleration $\mathbf{f}_{\mathrm{SRP}}=-\left(F S_{\perp} / m c\right)\left(1+\frac{4}{9} A\right) \mathbf{n}_{0}$ with the cross-section $S_{\perp}=\pi D^{2} / 4$ (e.g., Vokrouhlický and Milani, 2000) and (ii) we represented the sphere with a polyhedron model of 7200 surface facets and used methods from Section 2. Both methods provided identical results which conveniently validates our general method implementation in the OrbFit software. We used $D=270 \mathrm{~m}$, bulk density $2 \mathrm{~g} / \mathrm{cm}^{3}$ and albedo ${ }^{7} A=0.2$. We find that

\footnotetext{
${ }^{4}$ We thus evaluate the fit-continuous model in the terminology of Giorgini et al. (2008).

${ }^{5}$ For instance, the current uncertainty in the semimajor axis determination is about an order of magnitude larger than the corresponding change due to radiation pressure (e.g., Dermott et al., 2001).

${ }^{6}$ Note the bulk density for Apophis is unknown. We choose this value by considering an analogy with (25143) Itokawa (e.g., Abe et al., 2006), which is an object of similar spectral type and about the same size as Apophis. Should another value of the bulk density apply, one may use a simple inverse proportional scaling of our result with this parameter.

7 The effective albedo in the bracket of the radiation pressure formula $\mathbf{f}_{\mathrm{SRP}}$ for a sphere is given by $A=\frac{9}{2} \int_{0}^{1} d \mu_{0}\left[\mu_{0} K_{1}\left(\mu_{0}\right)+\sqrt{1-\mu_{0}^{2}} K_{2}\left(\mu_{0}\right)\right]$ for a general scattering law. Because $K_{1}\left(\mu_{0}\right)$ is reasonably well approximated by $\frac{2}{3} \mu_{0} A_{B}$ and is larger than $K_{2}\left(\mu_{0}\right)$ (see Fig. 2), we obtain $A \simeq A_{B} \simeq 0.2$ where this value of Bond albedo holds for the Hapke parameters chosen later in this section.
}

the closest-approach distance ${ }^{8}$ in April 13, 2029 has been shifted by about $4 \mathrm{~km}$ with respect to the nominal value $38046.825 \mathrm{~km}$. This result is in a good agreement with a similar test reported by Giorgini et al. (2008). Moreover, it shows the effect of direct radiation pressure $\mathbf{f}_{\text {inc }}$ is fairly small compared to the Yarkovsky effect, contrary to the finding of Rubincam.

\subsection{Rubincam's hemispheric model}

Next, we numerically verified conclusions from the analytic model of Rubincam (2007) and, at the same time, further tested implementation of the radiation forces in the OrbFit software. We constructed a hemispheric model using 7200 triangular surface facets. We oriented the pole position of the hemisphere in the orbital plane of Apophis with an arbitrary tilt $\beta$ from the direction to pericenter and ran several simulations. In order to directly compare our results with those of Rubincam, we considered the reflected component of the radiation only using the Lambertian sunlight reflection and set the albedo equal to unity. The diameter of the hemisphere was $270 \mathrm{~m}$ and the bulk density $2.8 \mathrm{~g} / \mathrm{cm}^{3}$ in this case. Extreme differences with respect to the nominal model were observed for $\beta=0^{\circ}$ and $\beta=90^{\circ}$. In the first case the effect was basically zero and in the second case the distance of the closest approach in April 13, 2029 has been shifted by $88 \mathrm{~km}$ with respect to its nominal value (maximum over all $\beta$ values). This is in a very good agreement with the analytic estimate obtained in Section 2.3. Adding the direct (incident) radiation pressure increases the effect by only $\sim 4 \mathrm{~km}$, in agreement with the previous test. If this were the true radiation effect it would be very significant after the assumed orbital improvements in 2013. While this effect seems large, we must recall that in reality the effect of radiation pressure may be only one-fifth to one-fourth of this maximum value, depending on the real albedo. A larger portion has to be moved to the thermal budget and can produce still larger orbital effect when a non-zero thermal inertia is taken into account.

\subsection{Gaussian random spheres used}

The hemispheric shape model used in Rubincam (2007) is clearly a gross idealization of real asteroid shapes. A much better statistical representation is provided by a sample of Gaussian random spheres with properly chosen parameters (see, e.g., Muinonen, 1998; Muinonen and Lagerros, 1998). In the next set of simulations we thus used 200 Gaussian random spheres constructed by Vokrouhlický and Čapek (2002) and Čapek and Vokrouhlický (2004). ${ }^{9}$ For each of the spheres, we considered 10 random pole orientations in space giving us 2000 individual simulations in a run. We used bulk density of $2 \mathrm{~g} / \mathrm{cm}^{3}$ and scaled all shape models such that their volume was equal to a sphere with a diameter of $270 \mathrm{~m}$. Note that the diameter of Apophis is uncertain at the $20 \%$ level and its density is unmeasured. However, due to the inversely proportional effect of these parameters, our results may be scaled as necessary to allow for this. While performing our tests with the values given above, size and density uncertainty may eventually be accounted for by assuming an additional $\simeq 40-50 \%$ uncertainty. We used two representative assumptions about the sunlight scattering on the surface: (i) the Lambertian model with albedo $A=0.2$ and (ii) a Hapke model with the following set of parameters: $w=0.5$, $g=-0.35, h_{s}=0.02$ and $B_{0}=0.97$, yielding the geometric albedo $A_{g}=0.34$. Obviously, the choice of the individual Hapke parameters

\footnotetext{
${ }^{8}$ Note that for Apophis the close approach distance uncertainty coincides very well with the 3D along-track orbital uncertainty (S. Chesley, personal communication).

${ }^{9}$ The full sample of the 200 Gaussian random spheres used in this work was described and shown in Čapek (2007), available through http://sirrah.troja.mff.cuni.cz/ davok/.
} 
is not unique and the one mentioned above corresponds to somewhat larger value of the $G$ parameter in the IAU $H-G$ system (see Verbiscer and Veverka (1995) for the transformation between the IAU $A_{g}$ and $H-G$ photometric parameters and the Hapke parameters). With a more typical $G \simeq 0.15$ value we would have: $w=0.34$, $g=-0.27, h_{s}=0.05$ and $B_{0}=1.98$, yielding approximately the same geometric albedo value. We have checked that this choice of the Hapke parameters provide about the same statistical results as the first set and in general the freedom in the choice of Hapke parameters does not change our conclusions.

Statistical results for displacement of the close approach distance in April 13, 2029, referred to its nominal value, are summarized in Figs. 3 and 4. The upper panels - (a) - in both figures show the composite effect of the incident and reflected radiation components, while the lower panels - (b) - show the effect of the reflected part only. We note that the overall orbit displacement due to the radiation pressure is small, in particular significantly smaller if compared to its maximum value derived for the hemispheric shape model. Clearly, the typically much higher symmetry between the north and south hemispheres in the Gaussian shapes, and we also believe in real asteroids, diminishes the possible orbital effect (see Vokrouhlický and Milani (2000) for the proof of the null effect in the case of the rotational spheroids).

For sake of interest, we point out that the $\sim(1-2) \mathrm{km}$ standard deviation from the $\simeq 4 \mathrm{~km}$ mean due to the radiation pressure is actually comparable to the perturbation due to the Poynting-Rob-

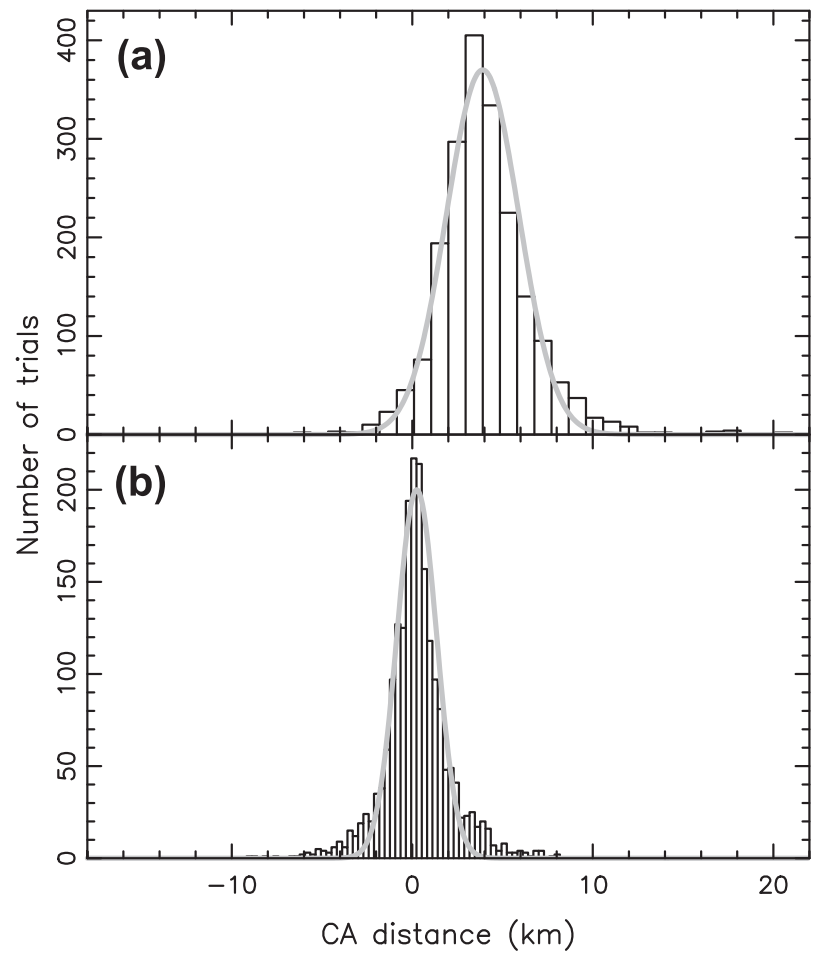

Fig. 3. Distribution of the close approach (CA) distance in April 2029 for 2000 tria simulations (200 different shapes modeled with Gaussian random spheres, each of which is given 10 different and random pole orientations) with the radiation forces. The close approach distance is referred to a nominal value $38046.825 \mathrm{~km}$ obtained in a simulation where radiation forces were excluded. Number of trials on the ordinate, close approach distance at the abscissa grouped into bins of $0.95 \mathrm{~km}$ (top) and $0.4 \mathrm{~km}$ (bottom) width. The top panel includes dynamical effects of the incident sunlight $\left(\mathbf{f}_{\text {inc }}\right)$ together with those of the reflected sunlight $\left(\mathbf{f}_{\mathrm{sca}}\right)$. We assume Lambertian model for the scattering with an albedo of 0.2 , effective size $D=270 \mathrm{~m}$ and bulk density $2 \mathrm{~g} / \mathrm{cm}^{3}$. The bottom panel shows effects of the reflected sunlight only. The grey Gaussian curves in both panels serve for a comparison only: the mean value at the top panel is $\sim 4 \mathrm{~km}$, corresponding to the effect of the incident radiation, and the standard deviations are $\sim 2 \mathrm{~km}$ (top) and $\sim 1.1 \mathrm{~km}$ (bottom). ertson (PR) component (e.g., Vokrouhlický and Milani, 2000). Indeed, the estimated secular change of the semimajor axis due to

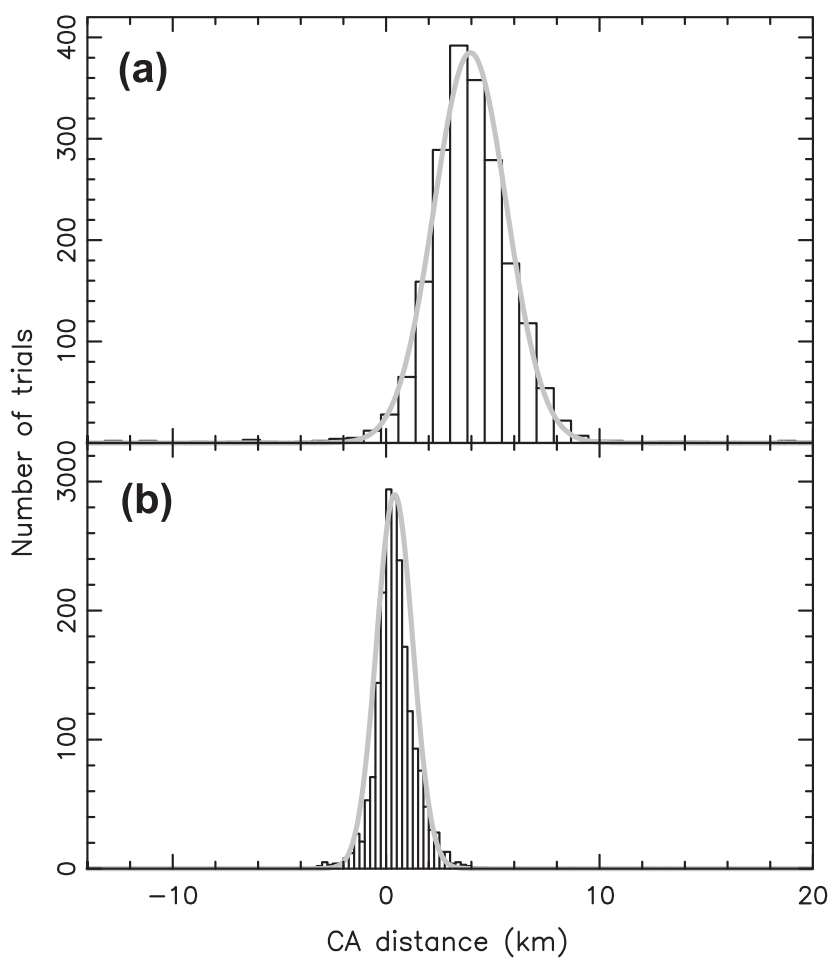

Fig. 4. The same as in Fig. 3, but now the reflected sunlight is modeled using the Hapke's bidirectional reflectance function (Section 2.2). The Hapke parameters are: $w=0.5, g=-0.35, h_{s}=0.02$ and $B_{0}=0.97$, yielding geometric albedo $A_{g}=0.34$. The grey Gaussian curves in both panels serve for a comparison only: the mean value at the top panel is $\sim 4 \mathrm{~km}$, corresponding to the effect of the incident radiation, and the standard deviations are $\sim 1.7 \mathrm{~km}$ (top) and $\sim 0.9 \mathrm{~km}$ (bottom).

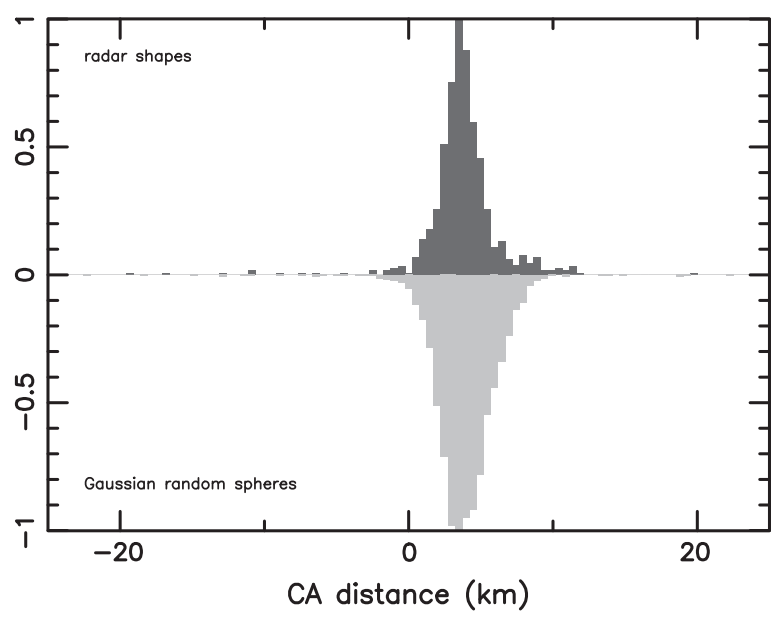

Fig. 5. Upper part: distribution of the close approach distance in April 2029 for 800 trial simulations (16 different radar-derived asteroid shapes, each of which is given 50 different and random pole orientations) with the radiation forces (ordinate values are arbitrarily normalized to unity in the maximum-occupied bin). The close approach distance is referred to a nominal value $38046.825 \mathrm{~km}$ obtained in a simulation where radiation forces were excluded. The sunlight reflected on the surface is modeled using the Hapke bidirectional function with parameters as in Fig. 4. Lower part (and inverted): normalized distribution of the close approach distance in April 2029 from the top panel (a) in Fig. 4 for comparison. Statistical properties of both distributions are basically identical: the difference in mean values is insignificant, while the standard deviation of the upper distribution $\sim 1.3 \mathrm{~km}$ is slightly smaller than that of the bottom distribution $\sim 1.7 \mathrm{~km}$. 
the PR effect for Apophis is $(d a / d t)_{\mathrm{PR}} \sim 3 \times 10^{-6} \mathrm{AU} / \mathrm{Myr}$, about a factor 100-500 smaller than the corresponding value due to the Yarkovsky forces. Including the PR acceleration into the OrbFit integrator we obtained $1 \mathrm{~km}$ displacement of the close approach distance in April 13, 2029.

\subsection{Radar shape models used}

While we believe the Gaussian random sphere shapes represent the true asteroid models rather well, we finally tested this assumption. For that purpose we downloaded 16 shape models of the near-Earth asteroids determined using the radar ranging and available from http://echo.jpl.nasa.gov/links.html. We used these shape models of real asteroids and re-ran our simulation choosing each time 50 possible and random pole orientation in space. We scaled all models to the Apophis' $270 \mathrm{~m}$ effective size, used $2 \mathrm{~g} / \mathrm{cm}^{3}$ bulk density and assumed Hapke model of the sunlight reflection with the above given set parameters.

Statistical results for the 2029 close-approach displacement, as referred to the nominal orbit, are shown in Fig. 5, where a comparison with the same simulation but Gaussian random sphere shape models is also shown. As expected, there is no fundamental difference between results for the Gaussian shapes and those where we used the real asteroid shapes.

\section{Conclusions}

Results from our work show that the orbital perturbation of Apophis due to the solar radiation pressure, as propagated to this asteroids' very close approach in April 2029 (critical for the further evolution of its orbit), is more then order of magnitude smaller than the perturbation due to the Yarkovsky effect. ${ }^{10}$ On the other hand, the few-kilometer size perturbation on the target plane position of the 2029 close approach of Apophis means the radiation pressure is a significant effect in absolute terms. This is because it can bring the true trajectory of Apophis close to or away from identified hundred-meter size (and smaller) keyholes associated with Earthimpacts in 2030s and later (see Chesley, 2006; Chesley et al., 2009). So two implications arise from our work.

First, until the Yarkovky effect for Apophis becomes constrained, the direct radiation pressure perturbation represents an addition to the orbital model that does not significantly improve its quality. Still, it can be included at low computer-time expense using the spherical model.

Second, things will change when the Yarkovsky effect is known for Apophis. Luckily, its close approach in January 2013 will at all likelihood provide a wealth of information: (i) not only physical parameters needed to the thermal force characterization will likely be constrained (such as the asteroid's shape, pole or thermal inertia), but (ii) the Yarkovsky effect could possibly also be directly measured using the precise orbital position as of 2013. Moreover, things may still further improve if high-quality astrometry data and physical parameter observations are taken in March 2021, during Apophis' next close approach to the Earth. At that moment, the Yarkovsky effect might be constrained to a significant-enough level that a more sophisticated model for direct solar radiation pressure might be included in the Apophis' orbital prediction. We believe this paper provides a suitable approach.

\footnotetext{
10 The strength of the Yarkovsky effect on Apophis is not known yet. Our claim is based on statistically typical value expected for a body of its size and orbit (see Chesley, 2006), and for the pole position distribution among near-Earth asteroids (e.g., Kryszczyńska et al., 2007).
}

\section{Acknowledgments}

This work has been partly supported by the Grant Agency of the Czech Republic (Grant 205/08/0064) and the Research Program MSM0021620860 of the Czech Ministry of Education. We thank Andrea Milani and an anonymous reviewer for important suggestions that helped to significantly improve the final version of the paper.

\section{References}

Abe, S., and 16 colleagues, 2006. Mass and local topography measurements of Itokawa by Hayabusa. Science 312, 1344-1349.

Behrend, R., Damerdji, Y., Reddy, V.V., Gary, B.L., Correia, H., 2005. (99942) 2004 MN4 combined light-curve. <http://obswww.unige.ch/\%7Ebehrend/ r099942a.png>.

Bottke, W.F., Vokrouhlický, D., Rubincam, D.P., Brož, M., 2002. Dynamical evolution of asteroids and meteoroids using the Yarkovsky effect. In: Bottke, W.F., Cellino, A., Paolicchi, P., Binzel, R.P. (Eds.), Asteroids, vol. III. Arizona University Press, Tucson, pp. 395-408.

Bottke, W.F., Vokrouhlický, D., Rubincam, D.P., Nesvorný, D., 2006. The Yarkovsky and YORP effects: Implications for asteroid dynamics. Annu. Rev. Earth Planet. Sci. 34, 157-191.

Čapek, D., 2007. Thermal Effects in Physics and Dynamics of Small Bodies of the Solar System. PhD Thesis, Charles University, Prague.

Čapek, D., Vokrouhlický, D., 2004. The YORP effect with finite thermal conductivity. Icarus 172, 526-536.

Chapman, C.R., 2004. The hazard of near-Earth asteroid impacts on Earth. Earth Planet. Sci. Lett. 222, 1-15.

Chesley, S.R., 2006. Potential impact detection for near-Earth asteroids: The case of 99942 Apophis $\left(2004 \mathrm{MN}_{4}\right.$ ). In: Lazzaro, D., Ferraz-Mello, S., Fernández, J. (Eds.), Asteroids, Comets, Meteors. Cambridge University Press, Cambridge, pp. 215228.

Chesley, S.R., Chodas, P.W., Milani, A., Valsecchi, G.B., Yeomans, D.K., 2002. Quantifying the risk posed by potential Earth impacts. Icarus 159, 423-432.

Chesley, S.R., Ostro, S.J., Vokrouhlický, D., Čapek, D., Giorgini, J.D., Nolan, M.C., Margot, J.-L., Hine, A.A., Benner, L.A.M., Chamberlin, A.B., 2003. Direct detection of the Yarkovsky effect by radar ranging to Asteroid 6489 Golevka. Science 302, 1739-1742.

Chesley, S.R., Vokrouhlický, D., Ostro, S.J., Benner, L.A.M., Margot, J.-L., Matson, R.L., Nolan, M.C., Shepard, M.K., 2008. Direct Estimation of Yarkovsky Accelerations on Near-Earth asteroids, Asteroids, Comets, Meteors 2008, Baltimore, Maryland, Contribution No. 1405, Paper Id. 8330.

Chesley, S.R., Milani, A., Tholen, D., Bernardi, F., Chodas, P., Micheli, M., 2009. An Updated Assessment of the Impact Threat from 99942 Apophis. DPS Meeting 41, \# 43.06.

Delbò, M., Cellino, A., Tedesco, E.F., 2007. Albedo and size determination of potentially hazardous Asteroids: (99942) Apophis. Icarus 188, 266-269.

Dermott, S.F., Grogan, K., Durda, D.D., Jayaraman, S., Kehoe, T.J.J., Kortenkamp, S.J., Wyatt, M.C., 2001. Orbital evolution of interplanetary dust. In: Grn, E., Gustafson, B.S., Dermott, S., Fechtig, H. (Eds.), Interplanetary Dust. SpringerVerlag, Berlin, pp. 569-640.

Giorgini, J.D., and 13 colleagues, 2002. Asteroid 1950 DA's encounter with Earth in 2880: Physical limits of collision probability prediction. Science 296, 132-136.

Giorgini, J.D., Benner, L.A.M., Ostro, S.J., Nolan, M.C., Busch, M.W., 2008. Predicting the Earth encounters of (99942) Apophis. Icarus 193, 1-19.

Hapke, B., 1981. Bidirectional reflectance spectroscopy. 1. Theory. J. Geophys. Res. 86, 3039-3054.

Hapke, B., 2002. Bidirectional reflectance spectroscopy. 5. The coherent backscatter opposition effect and anisotropic scattering. Icarus 157, 523-534.

Helfenstein, P., Veverka, J., 1989. Physical characterization of asteroid surfaces from photometric analysis. In: Matthews, M.S., Binzel, R.P., Gehrels, T. (Eds.), Asteroids, vol. II. Arizona University Press, Tucson, pp. 557-593.

Kryszczyńska, A., La Spina, A., Paolicchi, P., Harris, A.W., Breiter, S., Pravec, P., 2007. New findings on asteroid spin-vector distributions. Icarus 192, 223-237.

Mihalas, D., 1978. Stellar Atmospheres. W.H. Freeman and Co., San Francisco.

Milani, A., Chesley, S.R., Sansaturio, M.E., Bernardi, F., Valsecchi, G.B., Arratia, O., 2009. Long term impact risk for (101955) 1999 RQ. Icarus 203, 460-471.

Muinonen, K., 1998. Introducing the Gaussian shape hypothesis for asteroids and comets. Astron. Astrophys. 332, 1087-1098.

Muinonen, K., Lagerros, J.S.V., 1998. Inversion of shape statistics for small Solar System bodies. Astron. Astrophys. 333, 753-761.

Rubincam, D.P., 2007. Orbital YORP and asteroid orbit evolution, with application to Apophis. Icarus 192, 460-468.

Verbiscer, A.J., Veverka, J., 1995. Interpretation of the IAU two-parameter magnitude system for asteroids in terms of Hapke photometric theory. Icarus $115,369-373$

Vokrouhlický, D., Bottke, W.F., 2001. The Yarkovsky thermal force on small asteroids and their fragments. Choosing the right albedo. Astron. Astrophys. 371, 350353.

Vokrouhlický, D., Čapek, D., 2002. YORP-induced long-term evolution of the spin state of small asteroids and meteoroids. Rubincam's approximation. Icarus 159, 449-467. 
Vokrouhlický, D., Milani, A., 2000. Direct solar radiation pressure on the orbits of small near-Earth asteroids: Observable effects? Astron. Astrophys. 362, 746755.

Vokrouhlický, D., Milani, A., Chesley, S.R., 2000. Yarkovsky effect on near-Earth asteroids: Mathematical formulation and examples. Icarus 148 , 118-138.
Vokrouhlický, D., Chesley, S.R., Matson, R.D., 2008. Orbital identification for Asteroid 152563 (1992 Bf) through the Yarkovsky effect. Astron. J. 135, 23362340 . 